\title{
Formation of $\mathrm{SrTiO}_{3}$ Bicrystals using Spark Plasma
}

\author{
Sintering Techniques
}

\author{
L.A. Hughes and K. van Benthem \\ Department of Materials Science, University of California, Davis, 1 Shield Ave, Davis, \\ California 95616
}

\begin{abstract}
In this study, strontium titanate bicrystals with a twist grain boundary were successfully formed using a spark plasma sintering apparatus. Diffusion bonding was accomplished at high pressure and fast heating, and provides a novel method to form bicrystals at lower temperatures and accelerated time scales compared to more established techniques. Scanning electron microscopy was used to quantitatively evaluate the degree of successful interfacial bonding. Atomic resolution imaging using transmission election microscopy techniques combined with spatially resolved electron energy-loss spectroscopy measurements confirm a clean and atomically abrupt interface free of secondary phases.
\end{abstract}

The electronic and mechanical properties that control the functionality of oxide ceramic devices, such as high voltage capacitors, are determined by the core structure of grain boundaries [1]. Bicrystals, formed by two single crystals manipulated into a selected orientation, represent the ideal model systems for fundamental investigations of these core structures [2]. For example, crystallographic orientation of adjacent grains impacts lattice distortions in the vicinity of the boundary plane when compared to bulk $\mathrm{SrTiO}_{3}$ (STO) perovskite structure and is correlated to segregation behavior [3]. Bicrystal formation thereby enables studying specific grain boundary 
configurations as a function of processing conditions, impurity segregation and dopant concentrations [4-10].

Diffusion bonding of STO bicrystals is achieved by manipulation of pressure, time, temperature, and bonding atmosphere. Temperature is the dominant variable in diffusion as small changes in temperature impact the kinetics of every bonding mechanism. Changes in pressure affect the contact area between two half-crystals via plastic deformation of surface projections as well as increases dislocation densities, aiding in cationic diffusion [11-13].

STO bicrystals are typically formed at pressures below $1 \mathrm{MPa}$ with a temperature range of $1400-1500^{\circ} \mathrm{C}$ and bonding times between $3-20$ hours $[3,6,7]$. One reported exception is a $5^{\circ}$ tilt boundary, bonded at $700^{\circ} \mathrm{C}$ for 20 hours with a pressure of $0.2 \mathrm{MPa}$ [9]. Hutt et al observed if pressure exceeds 10MPa during bicrystal formation, instantaneous fracture of STO occurs [14].

The SPS apparatus, unlike other diffusion bonding techniques, uses a pulsed direct current, enabling fast heat rates, and applies forces above $3 \mathrm{kN}$. Considerable research efforts are dedicated to determining the mechanisms which lead to densification of powder compacts and grain growth during SPS $[15,16]$. Limited information is available on the influence of electric fields on grain boundary formation and resulting core structures [17, 18]. Forming bicrystals using similar experimental procedures as SPS will allow for systematic studies of grain boundary formation under applied electric fields.

In this paper, we report successful formation of STO bicrystals by SPS apparatus coupled with systematic determination of bonding parameters to maximize bonded interface quality. Atomic configuration, chemistry, and local electronic structure of formed grain boundary cores were characterized by high resolution transmission electron microscopy (HRTEM) and scanning 
transmission electron microscopy (STEM). Single crystals of STO with polished (100) surfaces (MTI Corporation) were sectioned into $5 \times 5 \mathrm{~mm}^{2}$ squares. These crystals were ultrasonically cleaned in acetone, isopropanol, and methanol and etched in buffered hydrofluoric acid $(\mathrm{pH}=4)$ to obtain $\mathrm{TiO}_{2}$-terminated (100) surfaces [19]. Two single crystals were fixed with misorientation angle $0^{\circ}, 4^{\circ}$, or $45^{\circ}$ around the $\langle 100>$ axis, placed in a cylindrical graphite die, and then positioned in a Dr. Sinter 5000 SPS apparatus (Sumitomo Coal Mining Co., Japan) (Fig.1). A 12-2 DC pulse sequence (12 seconds on, 2 seconds off) was applied with a pulsed bias of $4 \mathrm{~V}$ and direct current of 550A. Uniaxial pressure of $140 \mathrm{MPa}$ was applied to minimize contact resistance and vacuum was held at $\sim 10 \mathrm{~Pa}$. Processing times with Dr. Sinter 5000 SPS apparatus are limited to 100 minutes, thereby requiring variations in heating rate. Heating rates of 40$50^{\circ} \mathrm{C} /$ minute were used for bonding times below 90 minutes, while heating rates of 70 $80^{\circ} \mathrm{C} /$ minute were applied for a bonding time of 90 minutes. A cooling rate of $50^{\circ} \mathrm{C} /$ minute was used for all experiments. After SPS processing, samples were re-oxidized and annealed at $1200^{\circ} \mathrm{C}$ for $100-140 \mathrm{hrs}$ in air.

Samples were cross-sectioned and polished by diamond lapping film as well as colloidal silica for scanning electron microscopy (SEM) and electron backscatter diffraction (EBSD) characterization. A FEI Scios dual-beamed focused ion beam (FIB) instrument was used to prepare cross-sectional TEM samples and a Fischione Nanomill at $900 \mathrm{eV}$ and $500 \mathrm{eV}$ was used to reduce surface damage. HRTEM images were acquired using JEOL JEM2500 SE operating at 200kV. Atomic resolution STEM characterization was done via high-angle annular dark field (HAADF) imaging and EELS acquisition by aberration corrected FEI TEAM 0.5 instrument operating at $80 \mathrm{kV}[20]$. 
Table 1 details diffusion parameters and ensuing fraction of the successfully bonded STO bicrystal interface. Bonding temperatures were selected considering the applied pressure of $140 \mathrm{MPa}$ and the plasticity of STO. STO exhibits a ductile-brittle transition at $727-837^{\circ} \mathrm{C}$ and a brittle-ductile transition at $1191-1227^{\circ} \mathrm{C}$. Below $700^{\circ} \mathrm{C}$ and above $1200^{\circ} \mathrm{C}$, stresses greater than $120 \mathrm{MPa}$ were reported, indicating pressure can exceed $10 \mathrm{MPa}$ at certain temperatures without brittle failure. For the $700-1200^{\circ} \mathrm{C}$ temperature interval, STO is brittle with minor deformability up to $200 \mathrm{MPa}$ at $800^{\circ} \mathrm{C}$ [21]. Given SPS apparatus constraints, bicrystal A was bonded at a temperature of $1200^{\circ} \mathrm{C}$ for 15 minutes to maximize diffusion bonding time. Extensive microcracking was observed after bonding and, during annealing, these micro-cracks led to brittle failure of the sample after 16 hours. Sample fragments were too small for polishing to enable SEM characterization. Fracturing of bicrystal A was not unexpected as its bonding temperature is within the brittle-ductile transition temperature range in which STO provides less than $0.5 \%$ ductility.

Bicrystals with a misorientation angle of $0^{\circ}$ were synthesized by altering bonding temperature to ascertain optimal parameters while limiting interfacial stress. Temperatures of $600^{\circ} \mathrm{C}$ and $700^{\circ} \mathrm{C}$ were chosen according to the temperature dependent plasticity behavior of STO.

In this study, successful bicrystal formation is defined by a bonded interface fraction greater than $50 \%$ of the total measured contact length. Successful bonding conditions are observed when the grain boundary is not resolved during SEM imaging. Local non-bonding conditions are identified by a dark image contrast at the boundary location. Colloidal graphite paint, used during SEM specimen mounting, diffuses between the two crystals due to capillary effects and is the source of this dark contrast. Bonded interface fractions were calculated by 
subtracting the Feret diameter of detected voids and the length of resolved dark contrasts along the boundary plane from the overall contact length.

Fig. $2 a$ and $2 b$ are SEM images at two different locations in bicrystal G. In Fig. 2a, a line of dark contrast is observed, signifying a non-bonded boundary. This boundary changes in width, indicating onset of void formation. Two areas display successful bonding, marked by arrow \#1 and \#2 in Fig. 2a. In Fig. 2b, no boundary line is detected, illustrating successful bonding at this area. The grain boundary location is inferred from the location of two highly faceted voids.

Formation of $0^{\circ}$ bicrystals by SPS methods was accomplished with a $95 \%$ successfully bonded interface and minimal micro-crack formation at temperatures of $600^{\circ} \mathrm{C}$ and $700^{\circ} \mathrm{C}$. Additional bicrystals were formed with nominal twist angles of $4^{\circ}$ and $45^{\circ}$. EBSD experiments revealed misorientation angles of $4.2 \pm 0.3^{\circ}$ for bicrystal $\mathrm{E}$ and $46.1 \pm 0.5^{\circ}$ and $44.4 \pm 0.1^{\circ}$ for bicrystals $\mathrm{F}$ and $\mathrm{G}$, respectively. The misorientation angle of bicrystal $\mathrm{E}$ was not measured due to its fragility preventing additional polishing required for EBSD.

Bicrystal D shows a bonded interface fraction 2.2 times larger than observed for bicrystal G. Both bicrystals were synthesized with identical bonding parameters except for misorientation angle and annealing time. Bicrystal $\mathrm{D}$, with a nominal misorientation of $4^{\circ}$, was annealed for 50 hours. Bicrystal G, with a nominal misorientation of $45^{\circ}$, was annealed for 140 hours. It is concluded annealing times greater than 50 hours at a temperature of $1200^{\circ} \mathrm{C}$ do not significantly impact diffusion bonding quality. Changing misorientation angles does impact diffusion bonding quality, as expected. High-angle twist bicrystals have a large structural mismatch between the half-crystals, leading to higher interfacial stress states during boundary formation. Such 
circumstances hinder cross-boundary diffusion and decrease interface bonding as observed in bicrystal G.

The bonded interface fraction increases with increasing temperature and time for bicrystals with nominal $45^{\circ}$ twist misorientation. Further analysis illustrates as time and temperature increase, the total non-bonded area decreases due to formation of additional voids $[12,13]$. Bicrystal $\mathrm{E}$ had successfully bonded interface fraction of less than $2 \%$ as its bonding temperature of $700^{\circ} \mathrm{C}$ was too low for sufficient diffusion to occur. At a higher bonding temperature of $800^{\circ} \mathrm{C}$, this bonded interface fraction increases by a minimum of $30 \%$ (cf. Table 1). Increasing bonding time from 20 to 90 minutes also increases bonded interface fraction by roughly $10 \%$, while reducing the non-bonded region by approximately $34 \%$.

Fig. 2c and 2d show TEM images of voids observed in samples D and G, respectively. Both voids show defined facets along the (110) and (100) planes indicating the energetic favorability of these two planes lead to a preferred void shape for all samples, regardless of misorientation [22-24].

Previous studies of bicrystal formation do not detail bonded interface fractions. Successful bonding has been defined by atomically resolved interfaces or measured by fracture testing. The latter defines successful bonding when sample fracture occurs in the bulk rather than along the boundary plane or by comparison of the fracture strength of bicrystal to single crystal [25-27]. Porosity is reported to occur at the boundary periphery or center [27-29], indicating bicrystal quality is not defined by the presence of pores. Bicrystals with a $45^{\circ}$ twist boundary were deemed not successful by the conservative definition utilized in this study. However, 
bicrystal fracture was observed only in the bulk, indicating bicrystal G, with a successfully bonded interface fraction of $45.8 \pm 0.3 \%$, is sufficiently bonded.

Figs. $3 \mathrm{a}$ and $3 \mathrm{~b}$ show HRTEM and HAADF-STEM micrographs of bicrystal G recorded in $\langle 110\rangle$ zone axis with an edge-on orientation for the interface plane. Fig. 3a reveals an atomically abrupt grain boundary with no secondary phase or amorphous intergranular film, which is confirmed by HAADF-STEM imaging. A high degree of ordering is observed with localized periodic distortions, potentially signifying screw dislocations at arrow \#1 and \#2 in Fig. 3b [30, 31]. These two highly localized areas have considerably darker contrast, indicating reduced HAADF intensity. Changes in HAADF intensity can be associate with broken channeling conditions, but can also scale approximately with $\mathrm{Z}^{2}$, indicating these areas of dark contrast may be deficient in $\mathrm{Sr}$ [31]. These areas are located where a $\mathrm{Sr}$ column is expected according to STO crystal structure in $\langle 110\rangle$ zone-axis orientation. A Sr deficient grain boundary is consistent with previous work detailing low-angle tilt bicrystals [4]. To guide the eye, Fig. 3c shows a magnified view of Fig. $3 \mathrm{~b}$ with an overlaid atomic structure model. The model represents STO half-crystals in $\langle 100\rangle$ and $\langle 110\rangle$ zone-axis orientation. A step in the boundary plane with a height of one unit cell is observed, see black arrow (Fig. 3b).

Spatially resolved EELS determined local chemical composition and electronic structure across the grain boundary plane in bicrystal G (Fig. 4). The crystal field splitting of $\mathrm{Ti}_{3} \mathrm{~L}_{3}$ and $\mathrm{L}_{2}$ edges reduced from $2.5 \mathrm{eV}$ in the bulk to $1.3 \mathrm{eV}$ in the grain boundary core (Fig. 4a). This reduction indicates a change in $\mathrm{Ti}$ coordination, i.e. a valence state shift from $\mathrm{Ti}^{4+}$ to $\mathrm{Ti}^{(4-\delta)+}$ at the boundary. The Ti valence state shift and the reduction in $\mathrm{O}$ K edge intensity, observed in Fig. 4b, is consistent with increasing oxygen vacancy concentration at the boundary core. In addition, relative intensity of pre-peak $\mathrm{A}$ in the $\mathrm{O} \mathrm{K}$ near-edge fine structure slightly decreases at the 
boundary core, consistent with previous EELS studies of grain boundaries in STO [32]. Overall, variations in integrated EELS intensities and changes in Ti coordination mimic results for lowangle twist bicrystals [31].

In conclusion, this study reports the first successful formation of STO bicrystals using SPS apparatus. Twist grain boundaries with $0^{\circ}, 4^{\circ}$ twist, and $45^{\circ}$ nominal misorientation angles were fabricated at unusually high pressures with moderate temperatures and accelerated times compared to hot-pressing techniques. TEM characterization revealed atomically abrupt interface configurations with no observed secondary phases. Grain boundary cores show increased oxygen vacancy concentration and reduced $\mathrm{Ti}$ coordination similar to the literature. In the future, bicrystal formation using SPS techniques will allow for systematic investigation of electric field and heating rate effects on specific grain boundary structures.

\section{Acknowledgements}

LH gratefully acknowledges financial support by an US National Science Foundation Graduate Research Fellowship under Grant No. 1148897. Electron microscopy characterization and SPS processing at UC Davis was financially supported by a University of California Laboratory Fee award (\#12-LR-238313). Work at the Molecular Foundry was supported by the Office of Science, Office of Basic Energy Sciences, of the U.S. Department of Energy under Contract No. DE-AC02-05CH11231.

\section{Reference}

[1] Y.-M. Chiang, W.D. Kingery, D.P. Birnie, Physical ceramics: principles for ceramic science and engineering, J. Wiley, 1997.

[2] T. Gemming, S. Nufer, W. Kurtz, M. Ruhle, Journal of the American Ceramic Society, 86 (2013) $581-589$.

[3] S. Hutt, O. Kienzle, F. Ernst, M. Ruhle, Zeitschrift fur Metallkunde, 92 (2001) 105-109.

[4] K. Takehara, Y. Sato, T. Tohei, N. Shibata, Y. Ikuhara, Journal of Materials Science, 49 (2014) $3962-3969$.

[5] N. Ichinose, H. Haneda, Journal of Electroceramics, 4 (1999) 7-13.

[6] T. Mitsuma, T. Tohei, N. Shibata, T. Mizoguchi, T. Yamamoto, Y. Ikuhara, Journal of Materials Science, 46 (2011) 4162-4168. 
[7] Y. Sato, T. Tanaka, F. Oba, T. Yamamoto, Y. Ikuhara, T. Sakuma, Science and Technology of Advanced Materials, 4 (2003) 605-611.

[8] M.M. McGibbon, N.D. Browning, M.F. Chrisholm, A.J. McGibbon, S.J. Pennycook, V. Ravikumar, V.P. Dravid, Science, 266 (1994) 102-104.

[9] Z. Zhang, W. Sigle, F. Phillipp, M. Rühle, Science, 302 (2003) 846-849.

[10] V. Ravikumar, V.P. Dravid, Ultramicroscopy, 52 (1993) 557-563.

[11] W.H. Rhodes, W.D. Kingery, Journal of the American Ceramic Society, 49 (1966) 521-526.

[12] O.M. Akselsen, Journal of Materials Science, 27 (1992) 569-579.

[13] A. Hill, E.R. Wallach, Acta Metallurgica, 37 (1989) 2425-2437.

[14] S. Hutt, University of Stuttgart, (2002).

[15] Z.A. Munir, U. Anselmi-Tamburini, M. Ohyanagi, Journal of Materials Science, 41 (2006) 763-777.

[16] J. Wang, H. Conrad, Journal of Materials Science, 49 (2014) 6074-6080.

[17] S.C. Byeon, K.S. Hong, Materials Science and Engineering: A, 287 (2000) 159-170.

[18] H. Conrad, D. Yang, Philosophical Magazine, 90 (2010) 1141-1157.

[19] K. Takahashi, A. Ohtomo, M. Kawasaki, H. Koinuma, Materials Science and Engineering: B, B41 (1996) 152156.

[20] C. Kisielowski, B. Freitag, M. Bischoff, H. van Lin, S. Lazar, G. Knippels, P. Tiemeijer, M. van der Stam, S. von Harrach, M. Stekelenburg, M. Haider, S. Uhlemann, H. Müller, P. Hartel, B. Kabius, D. Miller, I. Petrov, E.A. Olson, T. Donchev, E.A. Kenik, A.R. Lupini, J. Bentley, S.J. Pennycook, I.M. Anderson, A.M. Minor, A.K. Schmid, T. Duden, V. Radmilovic, Q.M. Ramasse, M. Watanabe, R. Erni, E.A. Stach, P. Denes, U. Dahmen, Microscopy and Microanalysis, 14 (2008) 469-477.

[21] S. Taeri, D. Brunner, W. Sigle, M. Ruhle, Zeitschrift fur Metallkunde, 95 (2004) 433-446.

[22] C. Herring, in W.E Kingston (Ed.), Physics of Powder Metallurgy McGraw-Hill, New York, (1950) 143-179.

[23] W.D. Kingery, B. Francois, In: S. Somiya and Y. Moriyoshi (Eds.) Sintering Key Papers, (1990) 467-486.

[24] W.C. Carter, A.M. Glaeser, Communications of the American Ceramic Society, (1984) C124-C127.

[25] K. Hartmann, R. Wirth, W. Heinrich, Physics and Chemistry of Minerals, 37 (2009) 291-300.

[26] S.J. Kim, H. Myoren, J. Chen, K. Nakajima, T. Yamashita, Cryogenics, 35 (1995) 901-903.

[27] K. Sato, H. Miyazaki, Y. Ikuhara, H. Kurishita, H. Yoshinaga, Materials Transactions, 31 (1990) 865-872.

[28] Q.D. Jiang, X.Q. Pan, J. Zegenhagen, Physical Review B, 56 (1997) 6947-6951.

[29] M. Dupeux, Journal of Crystal Growth, 66 (1984) 169-178.

[30] Z. Zhang, W. Sigle, W. Kurtz, Physical Review B, 69 (2004) 144103-144101-144107.

[31] L. Fitting, S. Thiel, A. Schmehl, J. Mannhart, D.A. Muller, Ultramicroscopy, 106 (2006) 1053-1061.

[32] M. Varela, J. Gazquez, S.J. Pennycook, MRS Bulletin, 37 (2012) 29-35. 
Table 1 Parameters for Diffusion bonding and the resulting bicrystal boundary properties.

\begin{tabular}{lllllllll}
\hline Bicrystal & $\begin{array}{l}\text { Twist } \\
\text { Orientation } \\
\left({ }^{\circ}\right)\end{array}$ & $\begin{array}{l}\text { Bond } \\
\text { Temperature } \\
\left({ }^{\circ} \mathrm{C}\right)\end{array}$ & $\begin{array}{l}\text { Bond } \\
\text { Time } \\
(\mathrm{min})\end{array}$ & $\begin{array}{l}\text { Anneal } \\
\text { Temperature } \\
\left({ }^{\circ} \mathrm{C}\right)\end{array}$ & $\begin{array}{l}\text { Anneal } \\
\text { Time } \\
(\mathrm{hrs})\end{array}$ & $\begin{array}{l}\text { Bonded } \\
\text { Interface } \\
\pm .3(\%)\end{array}$ & $\begin{array}{l}\text { Void } \\
\text { Length } \\
\pm .3(\%)\end{array}$ & $\begin{array}{l}\text { Un- } \\
\text { bonded } \\
\pm .3 \text { Area }\end{array}$ \\
$\mathrm{A}$ & $0^{*}$ & 1200 & 15 & 1200 & 16 & -- & -- & -- \\
$\mathrm{B}$ & 0 & 600 & 90 & 1200 & 100 & 92.3 & 7.3 & -- \\
$\mathrm{C}$ & 0 & 700 & 90 & 1200 & 100 & 99.7 & .003 & -- \\
$\mathrm{D}$ & $4.3 \pm .3$ & 800 & 20 & 1200 & 50 & 79.2 & 11.1 & 9.7 \\
$\mathrm{E}$ & $45^{*}$ & 700 & 60 & 1200 & 140 & 1.3 & .6 & 98.1 \\
$\mathrm{~F}$ & $46.1 \pm .5$ & 800 & 20 & 1200 & 140 & 35.4 & 13.4 & 51.2 \\
$\mathrm{G}$ & $44.4 \pm .1$ & 800 & 90 & 1200 & 140 & 45.8 & 36.6 & 17.6 \\
\hline
\end{tabular}




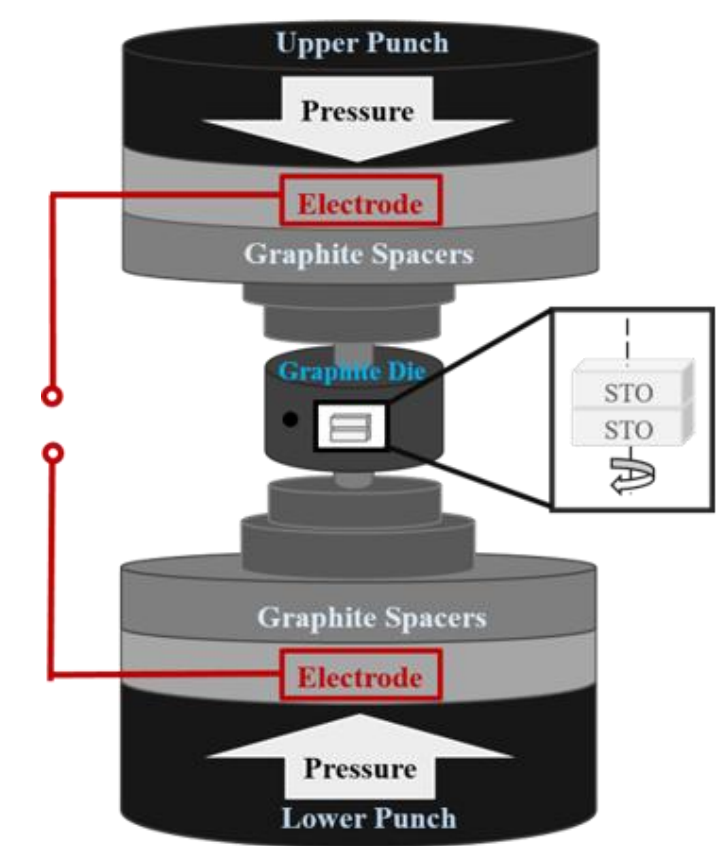

Fig 1 Experimental setup of the SPS

apparatus

.

$$
\text { mental setup of the SPS }
$$

Pressure$$
\text { (2) }
$$ 

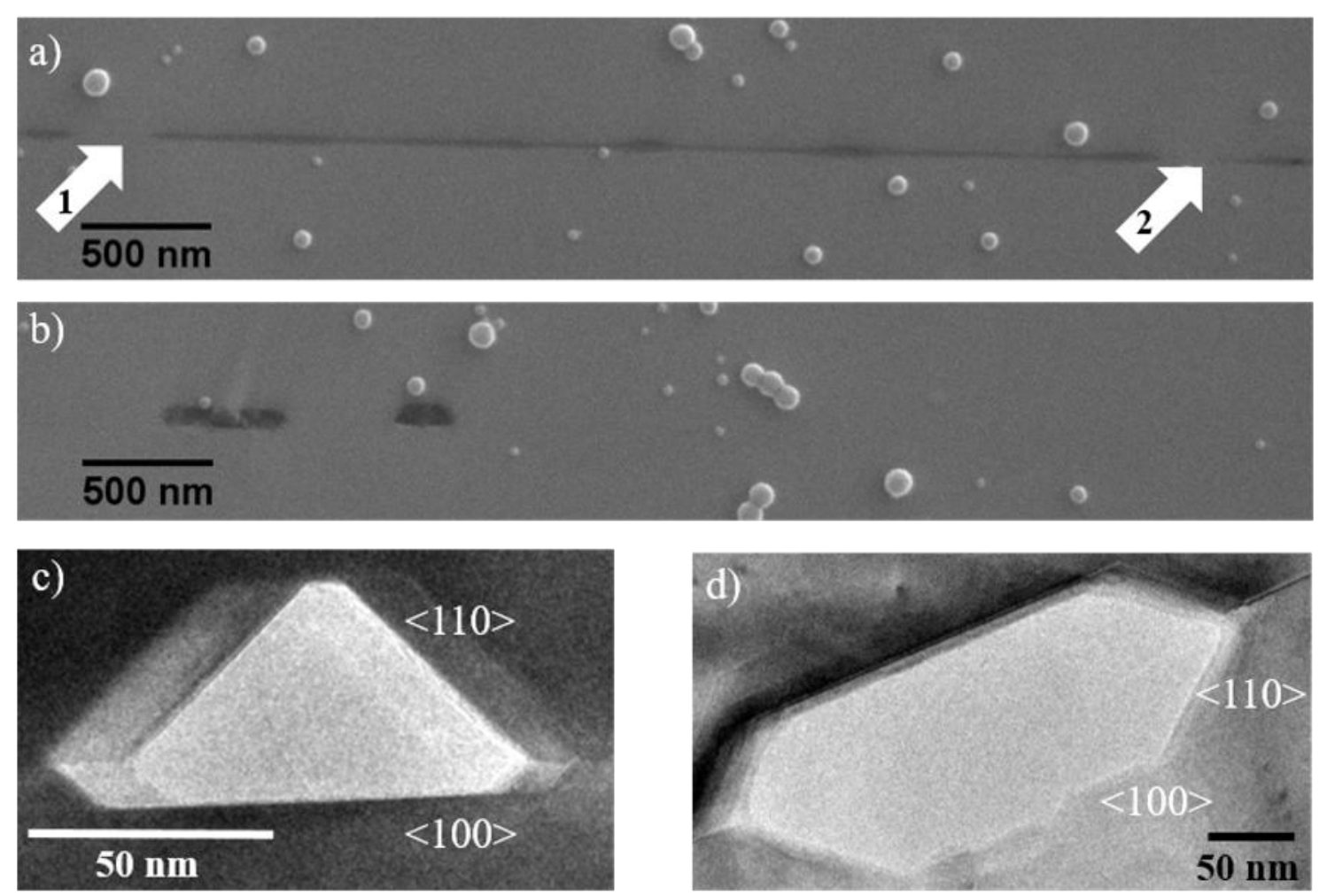

Fig. 2 Voids observed at different bicrystal boundaries (a) SEM image of bicrystal G with noticeable boundary line and voids. (b) SEM image of Bicrystal $\mathrm{G}$ with visible voids, but boundary line is not visible. (c) and (d) are TEM bright field images of voids observed at the boundary in Bicrystal D and Bicrystal G, respectively 


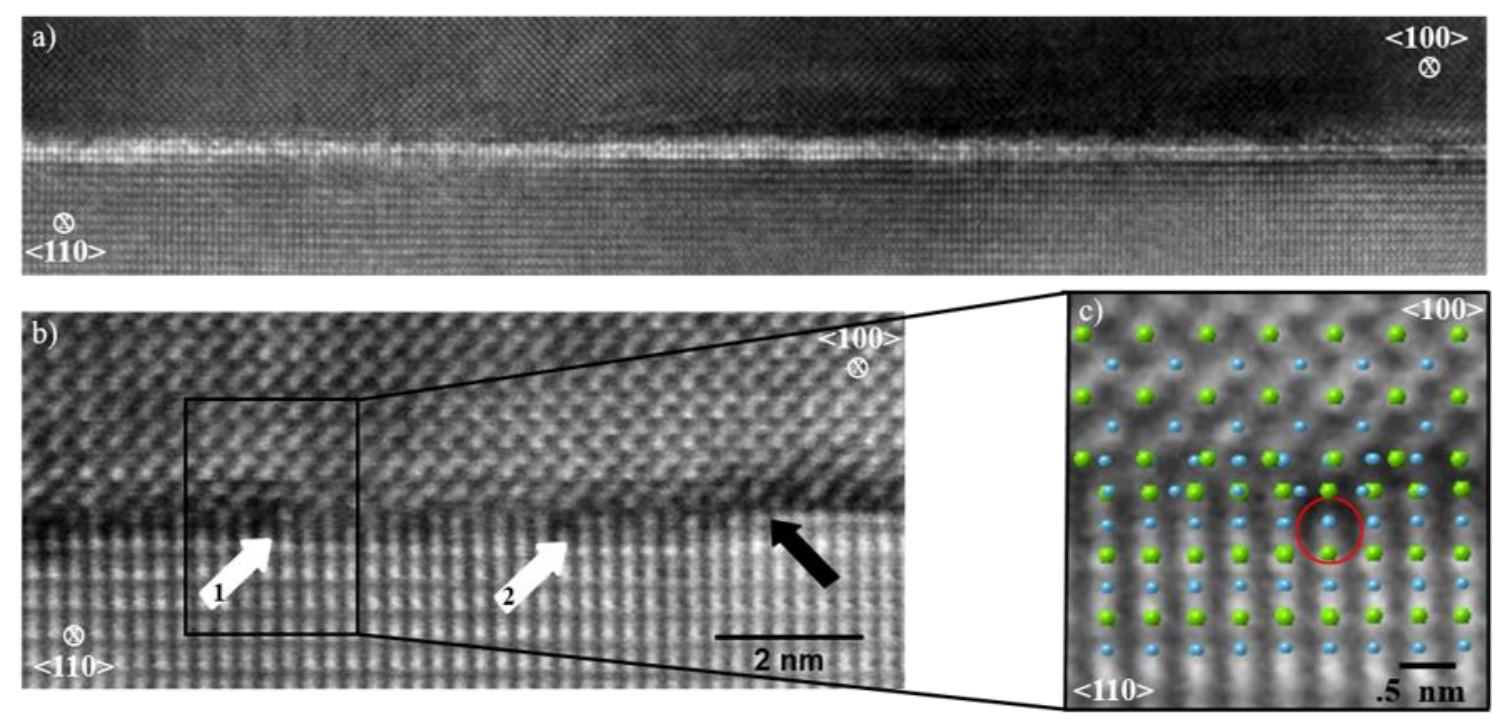

Fig. 3 (a) HRTEM image of the bicrystal boundary G. (b) HRSTEM DF image of the same boundary. (c) Simulated structure of a $45^{\circ}$ bicrystal boundary created in CrystalMaker® 

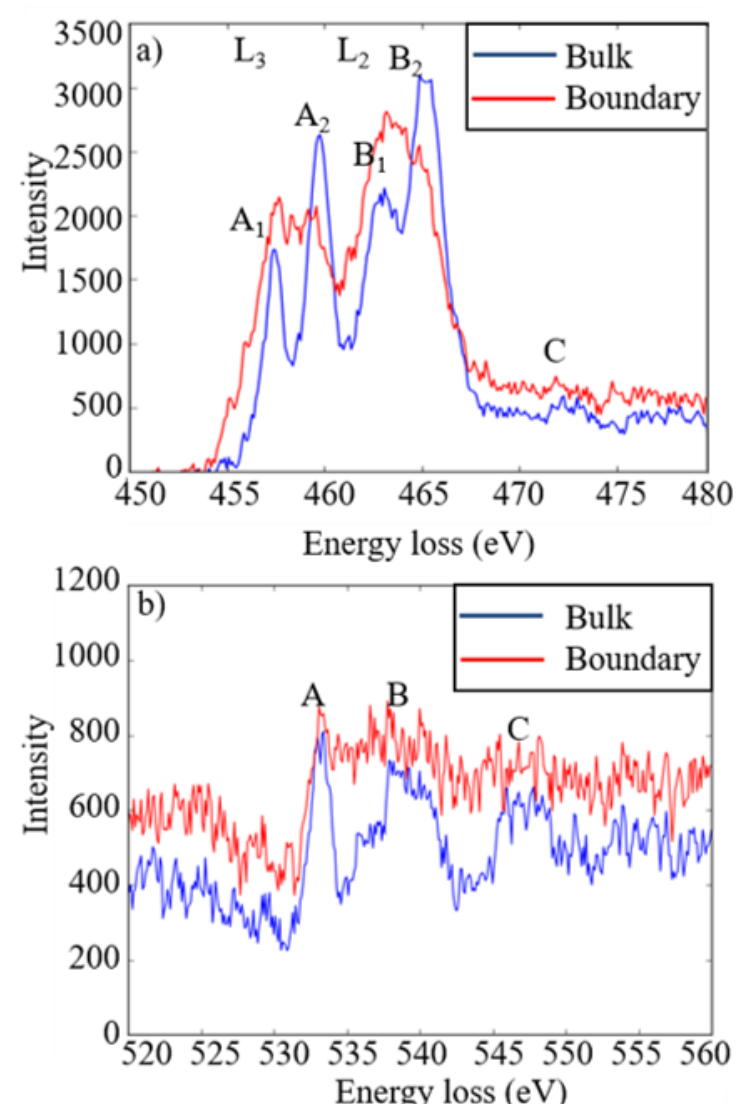

Fig. 4 EELS spectra, acquired at the

bicrystal boundary and in the bulk,

showing the near edge fine structure of

(a) the $\mathrm{Ti} \mathrm{L}_{2,3}$ edge and (b) the $\mathrm{O} \mathrm{K}$

edge. 